\title{
New Downlink Scheduling Framework for Hybrid Unicast and Multicast Traffic in WiMAX Networks
}

\author{
Rashid Karimi $^{\text {a, }}{ }^{* 1}$, Saleh Yousefi ${ }^{\mathrm{b}, * 2}$, Ali Azarpeyvand ${ }^{\mathrm{c}, * 3}$, Vahid.K.Alilou ${ }^{\mathrm{d}, * 4}$ \\ ${ }^{a}$ Department of Computer Engineering, Islamic Azad University of Zanjan, Znjan, Iran \\ ${ }^{b}$ Department of Computer Engineering, University of Urmia, Urmia, Iran \\ ${ }^{c}$ Department of Computer Engineering, University of Zanjan, Zanjan, Iran \\ ${ }^{d}$ Department of Computer Engineering, Science and Research Branch, Azad University, Tehran, Iran
}

\begin{abstract}
WiMAX networks based on IEEE 802.16 standard has expedited broadband wireless access surge in recent years. The traffic in these networks is identified in four types of class of service with different QoS requirements. Therefore, scheduling mechanism to manage these services in order to meet QoS requirements is a crucial fact and an important challenge. In this paper, for PMP mode of WiMAX networks, a two-level scheduling mechanism in MAC layer of Base Station (BS) has been proposed. The proposed scheduling algorithm takes into account hybrid unicast and multicast downlink traffic including three classes of service: rtps, nrtps and BE. In the first level of this scheduling mechanism, we have used the scheduling algorithms WRR and FCFS to schedule the connections and in its second level, the PQ algorithm based on Aging method is used to manage and schedule the packets. The functionality of the proposed scheduling algorithm is compared with priority queuing (PQ) algorithm. The resulting outcome of simulation shows that the proposed design has quite a better performance for Best Effort (BE) service class. Furthermore the delay of the rtps class and total throughput of the network is increased noticeably.
\end{abstract}

Index Terms: WiMAX Network; Scheduling; PQ Algorithm; Aging Method

(C) 2012 Published by MECS Publisher. Selection and/or peer review under responsibility of the Research Association of Modern Education and Computer Science

\section{Introduction}

In 2004, the IEEE 802.16 standard introduced a network called WiMAX and in 2005, its improved standard known as IEEE 802.16e was proposed and its resulting network called mobile WiMAX was introduced. [1] The IEEE 802.16 standard functions in the frequency limit of 66-10 GHZ and bandwidth of $10 \mathrm{Mbps}$ and covers a geographical area with the radius of 30 miles. The WiMAX network supports two topologies of Pointto-Multipoint(PMP) and Mesh mode. In the PMP mode, the subscribers stations (SSs) can only connect with each other through the Base Station (BS), but in the Mesh mode, in addition to this type of connection, the

* Corresponding author:

| E-mail address: ${ }^{* 1}$ Karimi.Rashid@Gmail.com; ${ }^{* 2}$ Syousefi@Urmia.ac.ir ${ }^{* 3}$ Azarpeyvand@ Gmail.com; ${ }^{* 4}$ AILab@ Srbiau.ac.ir 
subscribers stations can connect with each other as well [1,2]. In Fig. 1, a representation of the WiMAX network in PMP mode is shown[3]. The physical layer of WiMAX is based on the OFDM to which OFDMA was added in the 2005 version.

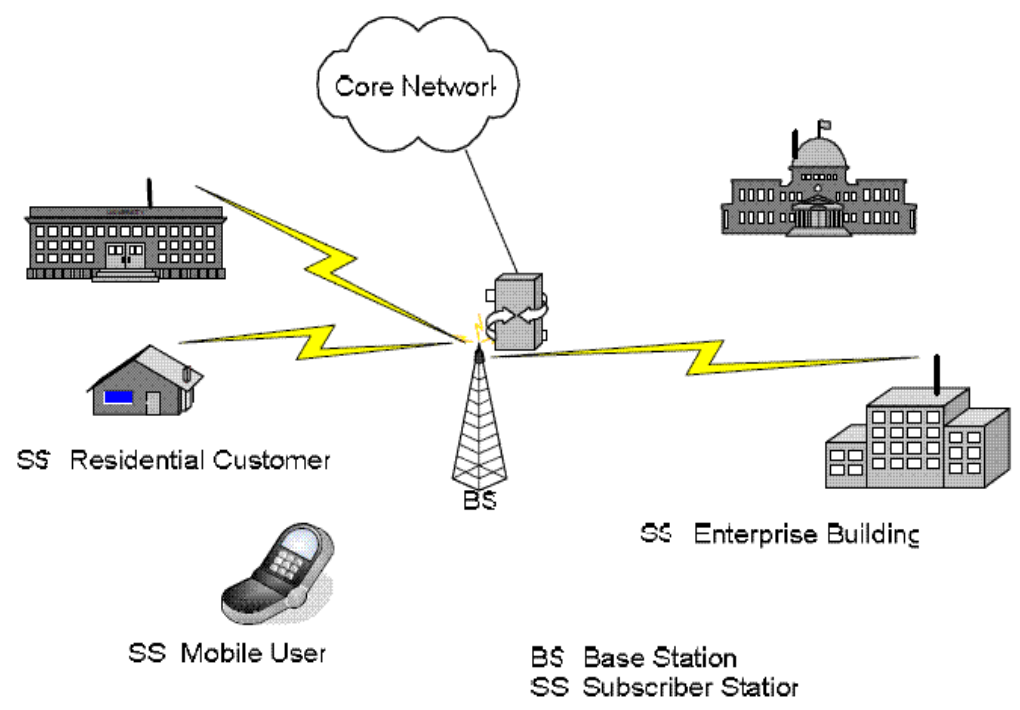

Fig. 1. An IEEE 802.16 network with PMP mode

The IEEE 802.16 standard, used two TDD and FDD duplexing methods to grant downlink and uplink bandwidths. When using FDD method, downlink and uplink channels function in different frequencies but at the same time, while in TDD method, uplink and downlink channels function in one frequency but at different times. Moreover, to give bandwidth, in addition to granting unsolicited bandwidth, the Base Station uses two types of polling services: unicast and multicast[2].

To support a great range of applications, WiMAX has defined four scheduling classes of service with different QoS requirements. These classes of service should be supported by the scheduler unit of Base Station. These classes of service are as follows: [4]

UGS: (unsolicited grant service), rtps: (real time polling service), nrtps: (none real time polling service),BE: (Best Effort). Of course, there is another scheduling service known as ertps which is added to the second version of the WiMAX which was introduced in 2005 and is based on UGS and rtps classes of services.

\section{Scheduling in WiMAX}

Scheduling is the most important element of MAC layer in WiMAX network, which guarantees the QoS requirements of different classes of service.[2]

There are three scheduling processes, two of which are in Base Station for uplink and downlink and the third one is for uplink in subscriber station [2]. In Base Station, the packets of higher layer are placed in different queues. Then based on QoS parameters and a series of extra information such as the channel condition, the downlink scheduler of the Base Station decides which queue should be served.

Since Base Station controls the access to transfer media, the second scheduler in Base Station, that is the uplink scheduler, decides about granting based on received requests of bandwidth from subscriber station and also considers the QoS parameters. Ultimately, the third scheduler is also in the subscriber station. The factors that may be taken into consideration for a scheduling designer's design are: QoS parameters, fairness, optimization of throughput, complexity of implementation and scalability. [2] 


\section{Related Work}

So far, many algorithms and scheduling mechanisms have been proposed regarding scheduling, some of which are basic algorithms and their other combined or developed mechanisms. Later, some of their instances will be pointed out.

In [5], Cheng et al. proposed a two- level scheduling design; the first level of this scheduler does the scheduling among the subscriber stations and in the second level, scheduling process is done for existing classes of service in subscriber stations. In [6], in order to optimize the DRR scheduling algorithm and a developed form of it, known as WDRR which is used for wireless environment, Sharma et al. have proposed a UF-DRR scheduling algorithm in which the fairness parameter of QoS requirement is optimized to an acceptable limit. In [7], Yi Wu et al. developed a scheduling format for WiMAX network and especially for BE class. In [8], Xiaojuan et al. who had developed a scheduling algorithm known as FEQ for WiMAX network in their earlier works, compared it with two other scheduling algorithms known as SP and DEPQ. In [9], Sayenko et al. proposed a comprehensive study on the issue of scheduling in WiMAX network which covers five classes of service in WiMAX. In [10], Mehrjoo et al. developed a scheduling design which, based on existing channel conditions and the background of each subscriber station. In [11], Yusheng ji et al. developed a scheduling design in focused form in Base Station which is composed of two levels of call admission control and scheduling unit.

\section{Proposed Design}

In our design, regardless of UGS class, we have defined and designed two scenarios. The first scenario is a scheduling structure under PQ scheduling algorithm which is considered the basic scenario and the second scenario is our proposed design which is a combined two-level scheduling mechanism based on the first scenario whose results are compared with the results of the first scenario.

\subsection{THE FIRST SCENARIO}

In this scenario, a downlink scheduler is designed in MAC layer of Base Station which works under PQ scheduling algorithm and schedules the received packets from subscriber station in downlink and only supports unicast polling mode. The structure of this scheduling framework is shown in Fig. 2.

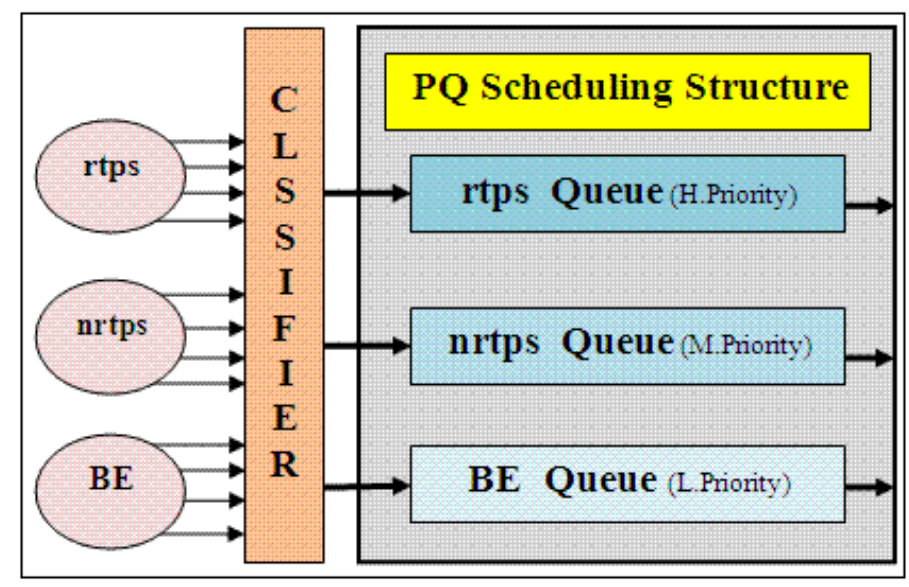

Fig. 2. PQ Scheduler Structure 
As it's shown in the figure, the existing packets in the connections of classes of service; rtps, nrtps and BE are classified in three priority queues with different sizes in a way that rtps queue has the highest priority and size and BE queue has the lowest priority and size. The policy of this scheduling format is that until serving is not finished in the queue with higher priority, the queue with lower priority cannot be served. Therefore, to follow this policy, the queues with lower priority may encounter starvation and unfairness and consequently we'll have low priority class of service packet drop especially in case of BE class of service.

\subsection{THE SECOND SCENARIO}

The second scenario is our proposed design which is a two-level scheduling mechanism based on the first scenario. In the first level of this mechanism, the scheduling operation is done among the connections of classes of service; rtps, nrtps, and BE. Here, the WRR algorithm is used to schedule the connection of classes of service rtps and nrtps; and the FCFS algorithm is used to schedule the connection of BE class of service. Since the QoS requirements of rtps class are prior in comparison with nrtps, then the $\mathbf{W}$ parameter (weight : the percentage of granted bandwidth) in WRR algorithm causes that rtps connections, compared to nrtps connection, have relative and local priority so that the negative impact of Aging method, which is imposed on rtps class, is somehow compensated. In the second layer of this scheduling mechanism the triplet play class packets are defined in a way that the priority of queues is reduced from top to bottom. In order to meet QoS requirements of low priority classes of service, in this level, we have used the Aging method that is usually utilized to schedule the active processes in operating system level in a way that the queue with low priority gives feedback to the high priority queue. If the packet waiting time is more than a defined limit, then the packet takes feedback from a low priority queue to the queue with high priority. This policy helps reduce starvation in case of low priority queues which, in turn, results in reduction of packet drop rate. The structure of this scheduling mechanism is shown in figure 3.

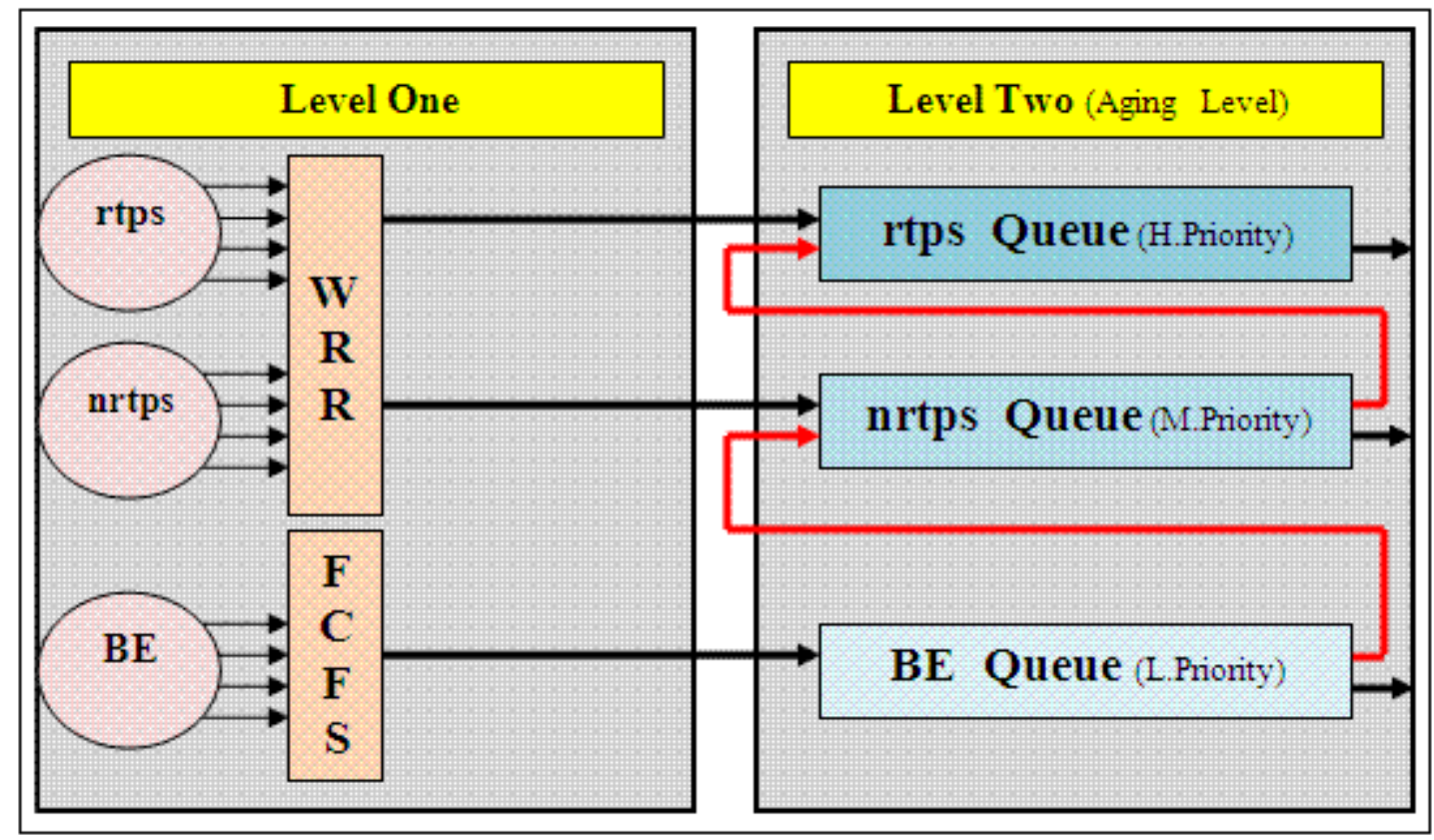

Fig. 3. Aging Scheduler Structure 
Moreover, in the second scenario, the unicast and multicast polling mechanisms have been applied to rtps class of service; in multicast polling, if a packet is solicited in the form of a solicitation from $\mathbf{N}$ subscriber station, then to answer this solicitation, instead of sending $\mathbf{N}$ packets, physically only one packet is sent to the soliciting group and quantitatively, in determining and assessing the throughput parameter, $\mathbf{N}$ packets are calculated. While in Unicast condition, in response to $\mathbf{N}$ solicitations from $\mathbf{N}$ subscriber stations, we should physically send $\mathbf{N}$ packets. Also, the packets that are solicited more, compared to other packets, have a higher priority in being sent and when implementing a multicast policy instead of a unicast policy, its superiority, compared to unicast mode, is noticeable in assessing QoS criteria such as throughput and delay because by implementing multicast mode, a remarkable percentage of released bandwidth and other classes of service find an opportunity to increase their throughput and consequently lower their delay.

\section{Simulation, Results and Comparison}

In the conducted simulation, we have considered one Base Station and five subscriber station in PMP (Pointto-Multipoint) mode. Subscriber stations can make connection of three types of classes of service and consequently send packets in the format of these connections to Base Station. The produced products are also scheduled in downlink by the Base Station scheduler after being sent to Base Station. In the simulation of this mechanism to approach the real status of a network, the packet generation has been based on the Poisson distribution, which results in a time period between the packet generation in the form of exponential distribution. Furthermore, the probability of generation classes of service packets is different and becomes buoyant regarding different assessment modes during simulation. The simulation parameters are shown in Table 1.

Table 1. Simulation Parameters

\begin{tabular}{ll}
\hline \multicolumn{1}{c}{ Parameter } & \multicolumn{1}{c}{ Value } \\
\hline Network Topology & PMP \\
\hline Number of BS & \multicolumn{1}{c}{5} \\
\hline Number of SS & Downlink \\
\hline Traffic Type & rtps.nrtps,BE \\
\hline Connection Types & 2 \\
Number of rtps Connection & 3 \\
Number of nrtps Connection & 4 \\
Number of BE Connection & $(2: 1)$ \\
\hline W in WRR(rtps : nrtps) & $1-5$ \\
\hline Min \& Max of N in Multicast & 160 Byte \\
rtps Packet Size & 1500 Byte \\
nrtps Packet Size & 1500 Byte \\
BE Packet Size & 30 Second \\
\hline Simulation Duration & \\
\hline
\end{tabular}

The results of simulation are as follows:

In Fig. 4, the average rtps traffic throughput is shown. In this figure, with the increase of packet generation, traffic also increases but because of the imposing impact of Aging method from the traffics with lower priority, the PQ scenario throughput overlaps with Aging scenario and the unicast or multicast in rtps traffic do not make a big difference because the multicast mode is only beneficial for throughput in BE and nrtps classes.

In Fig. 5, the average of rtps traffic delay is shown. In this figure, delay in aging scenario in unicast mode, as a result of imposing impact of Aging method, is more than the PQ scenario and as it is obvious, the impact of 
multicast mode on delay reduction is remarkable in comparison with unicast mode in Aging and PQ scenarios. Furthermore, rtps traffic has no packet drop in any of the three defined modes.

In Fig. 6, the average of nrtps traffic throughput is shown. In this figure, the nrtps traffic throughput in Aging scenario, because of imposing Aging method from BE queue and the negative impact of WRR algorithm in the scheduling first level on nrtps class, is less than the PQ scenario, but according to the figure, the traffic rate is more in multicast mode compared to unicast.

In Fig. 7, the average of nrtps traffic delay is shown which, due to mentioned reasons for Fig. 6, delay in Aging scenario is more than PQ scenario. However, the delay in multicast mode is less than the delay in unicast mode.

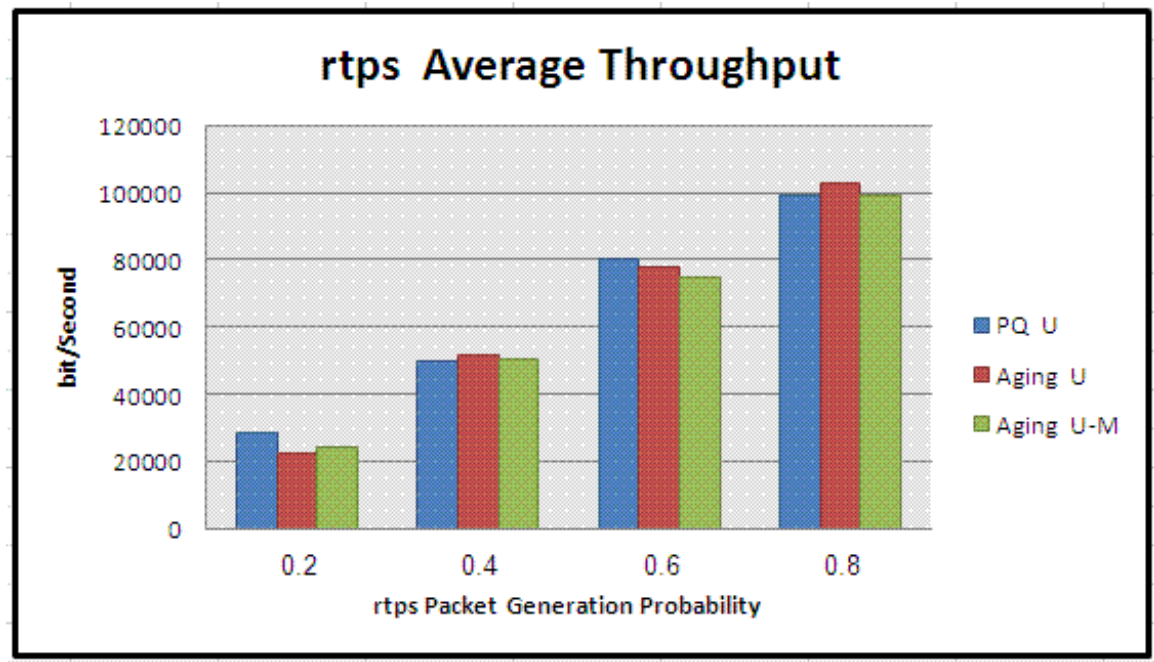

Fig. 4. Average Throughput in rtps Class

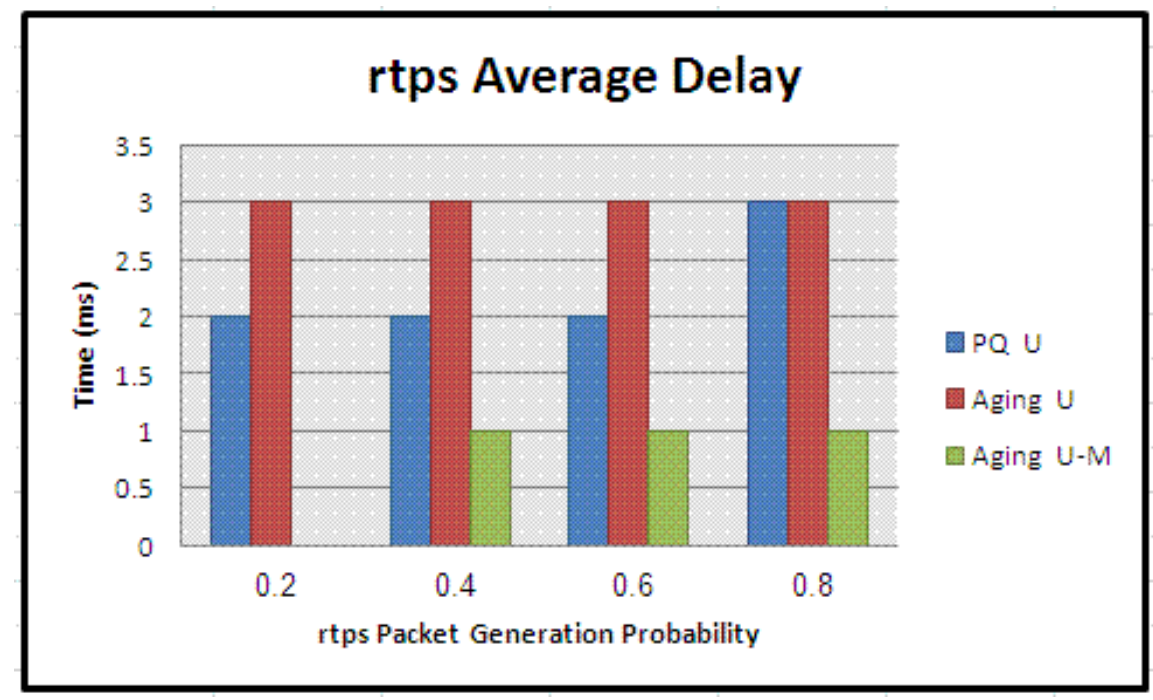

Fig. 5. Average Delay in rtps Class 


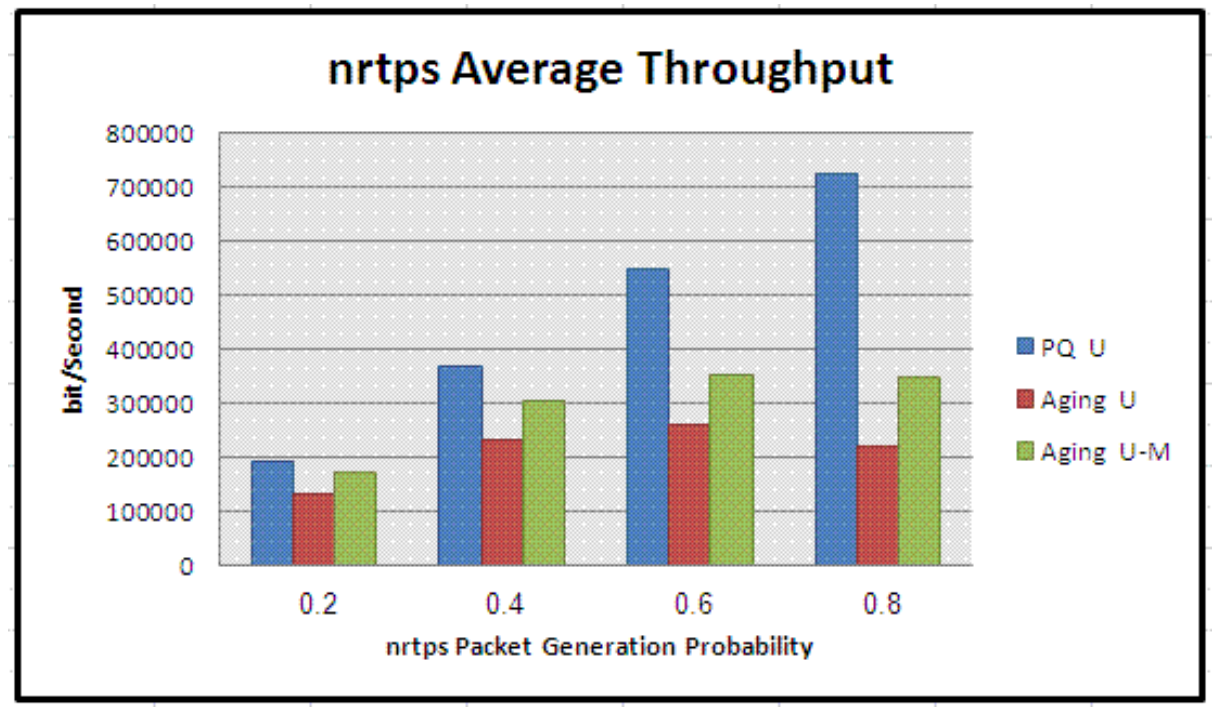

Fig. 6. Average Throughput in nrtps Class

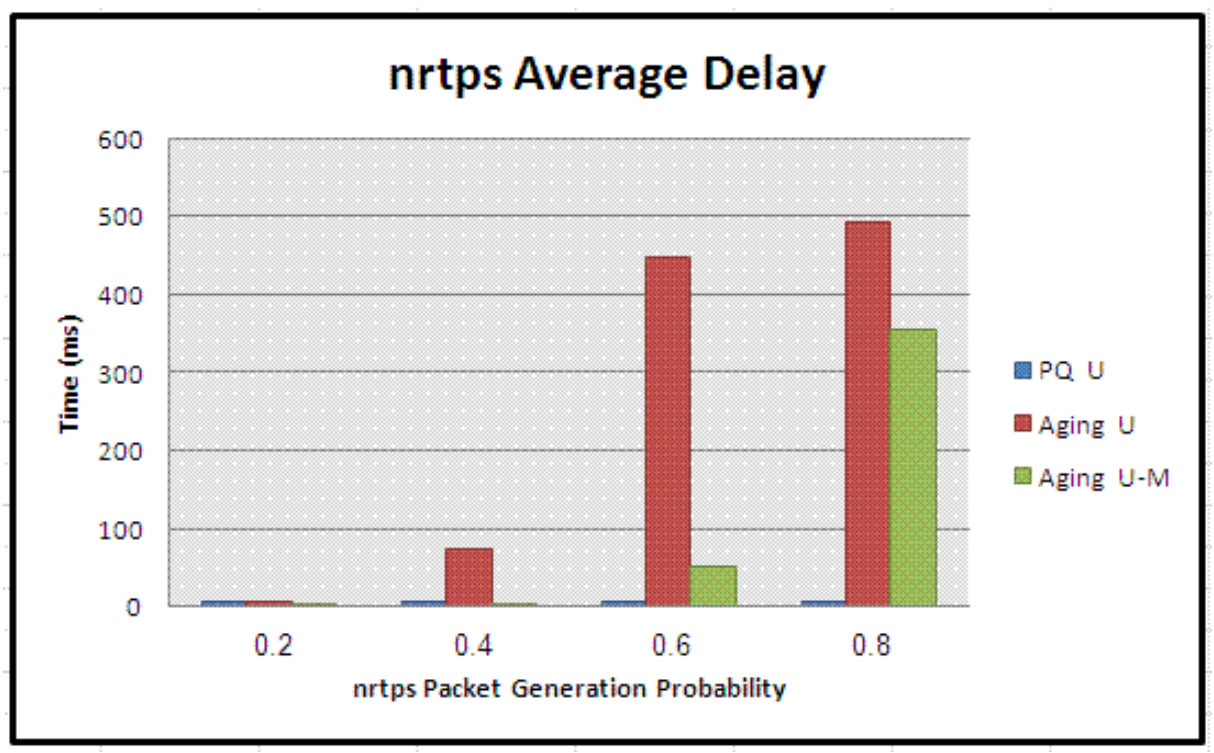

Fig. 7. Average Delay in nrtps Class

Unfortunately, in Fig. 8, in nrtps high traffic, in Aging scenario multicast and unicast modes, we notice packet drop which is lower in multicast mode compared to unicast mode.

In Fig. 9, the average of BE traffic throughput is shown, which in PQ scenario, due to BE traffic low priority, there is actually lower throughput than in Aging scenario which is absolutely obvious in high traffic. In Aging scenario, the multicast mode, compared to unicast mode lets a greater number of packets be sent and increases the throughput. 
In Fig. 10, average of BE traffic delay is shown, which due to the reasons mentioned about Fig. 9, delay in PQ scenario is a lot more than Aging scenario, and in multicast mode, compared to unicast mode, delay rate is less.

In Fig. 11, BE packet drop rate is shown that, because of mentioned reasons for Fig. 9 and Fig. 10, in PQ scenario and with the possibility of increase in traffic, this parameter also increases.

Finally, in Fig. 12, the average of the throughput rate for the whole system is shown. As it is clear, with the increase of probability rate of generation rtps packets, the advantage of multicast to unicast mode becomes even more evident. Because in this case, the rtps sending traffic decreases and consequently, BE and nrtps classes use the emptied spaces of bandwidth and find more opportunity to send the packet compared to unicast mode and consequently the average throughput of the system becomes more than the unicast mode.

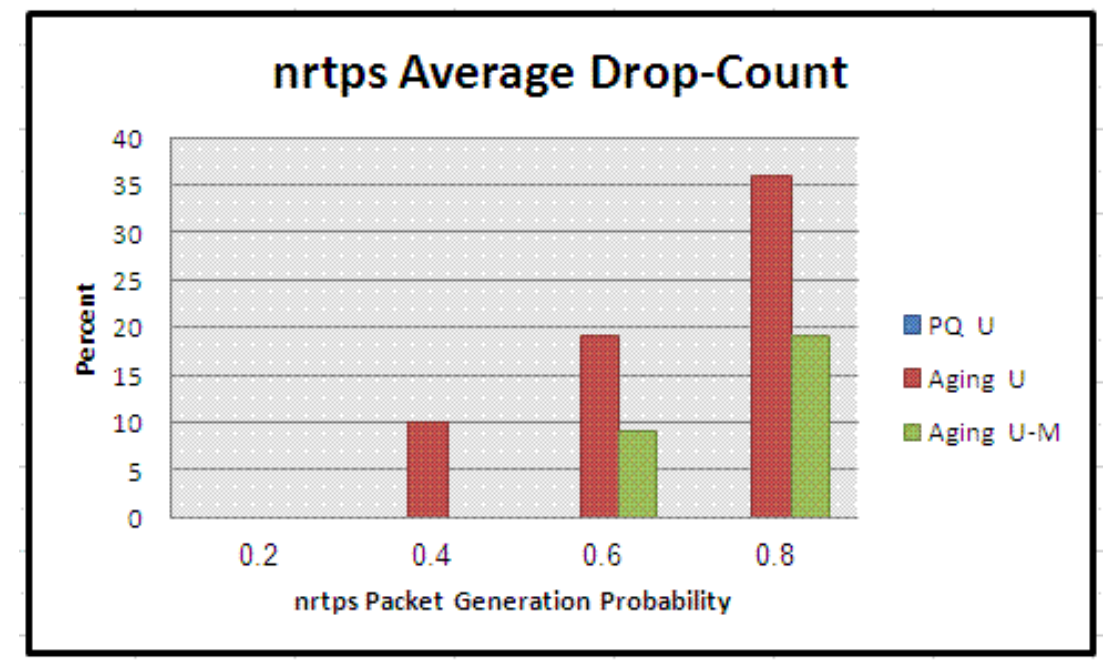

Fig. 8. Average Drop-Count in nrtps Class

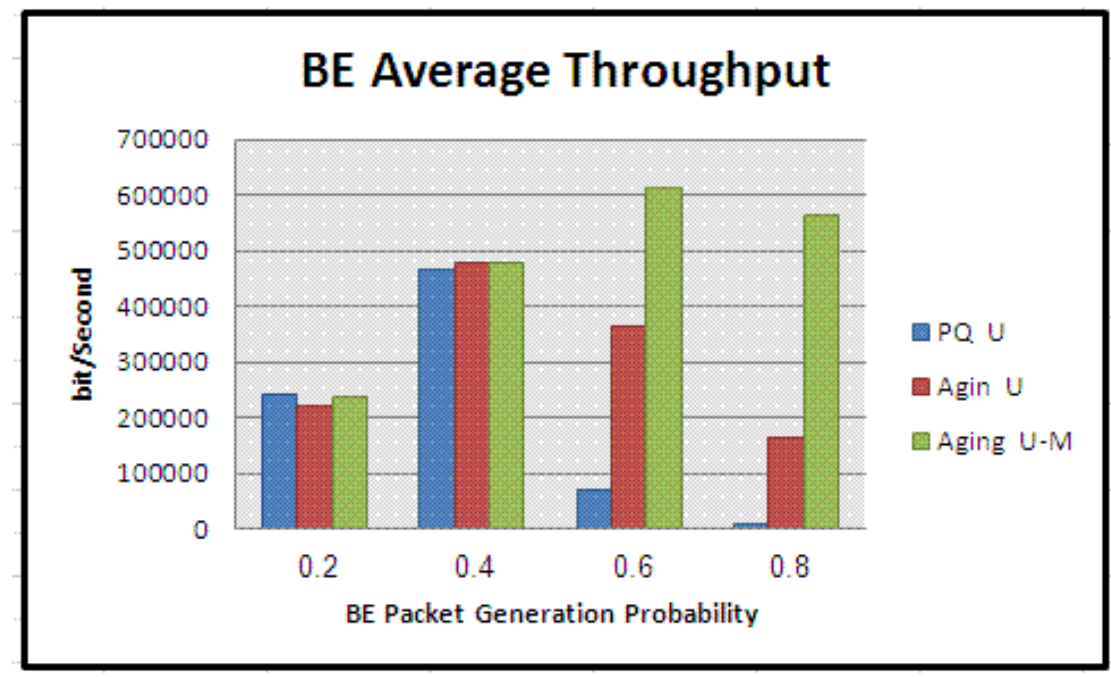

Fig. 9. Average Throughput in BE Class 


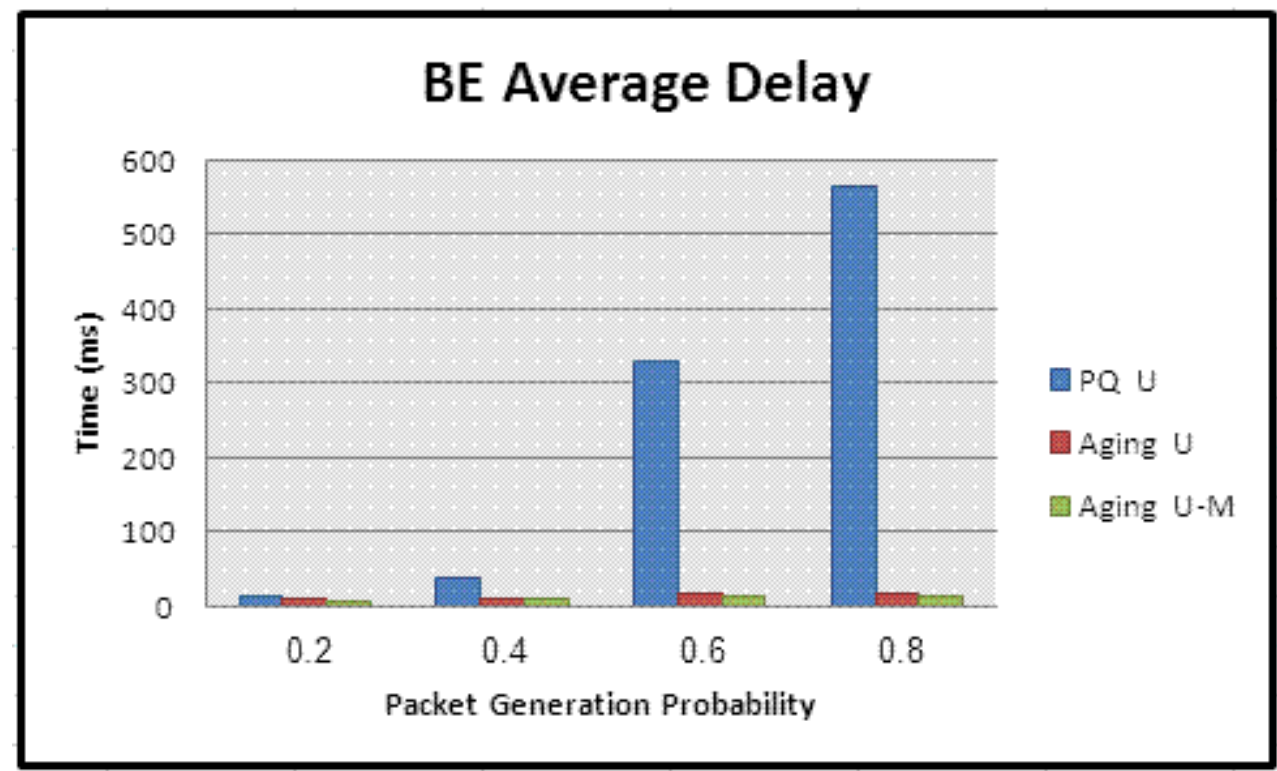

Fig. 10. Average Delay in BE Class

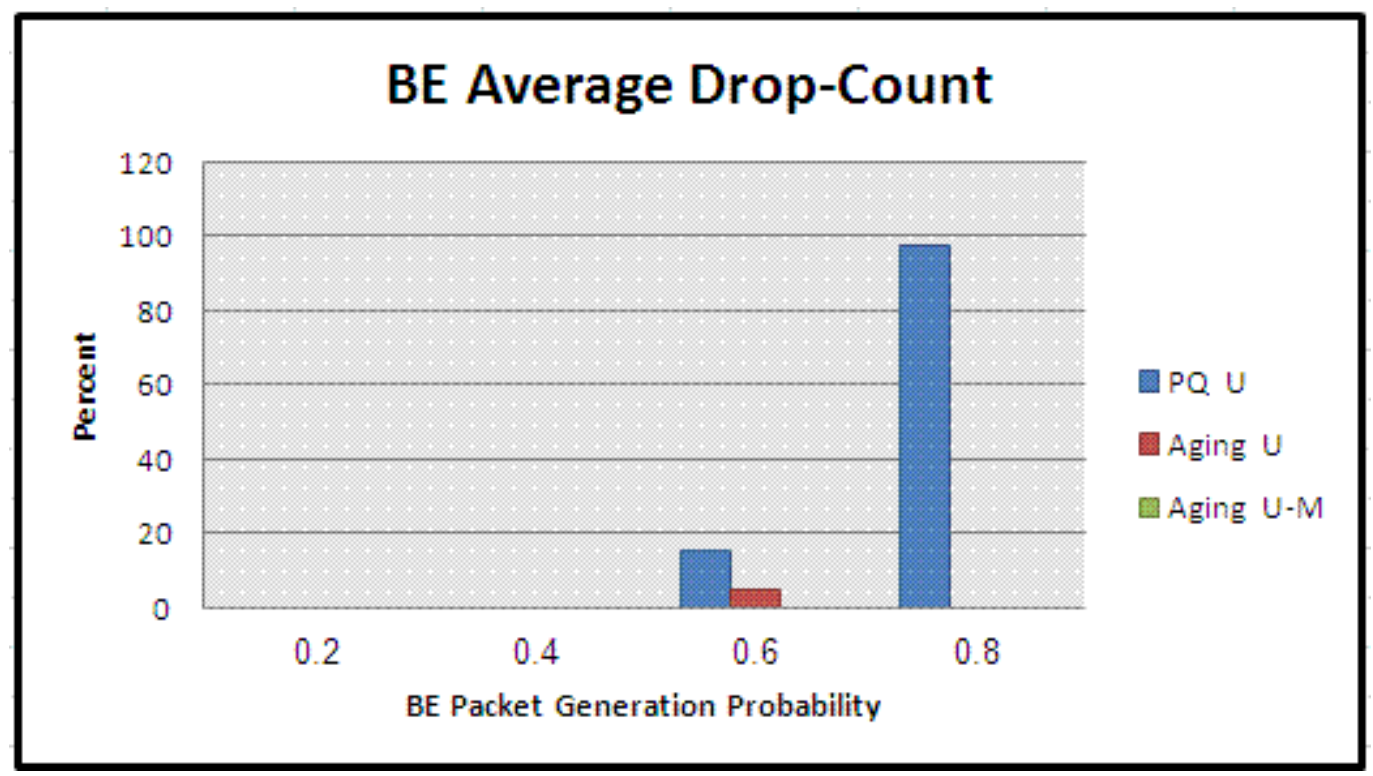

Fig. 11. Average Drop-Count in BE Class 


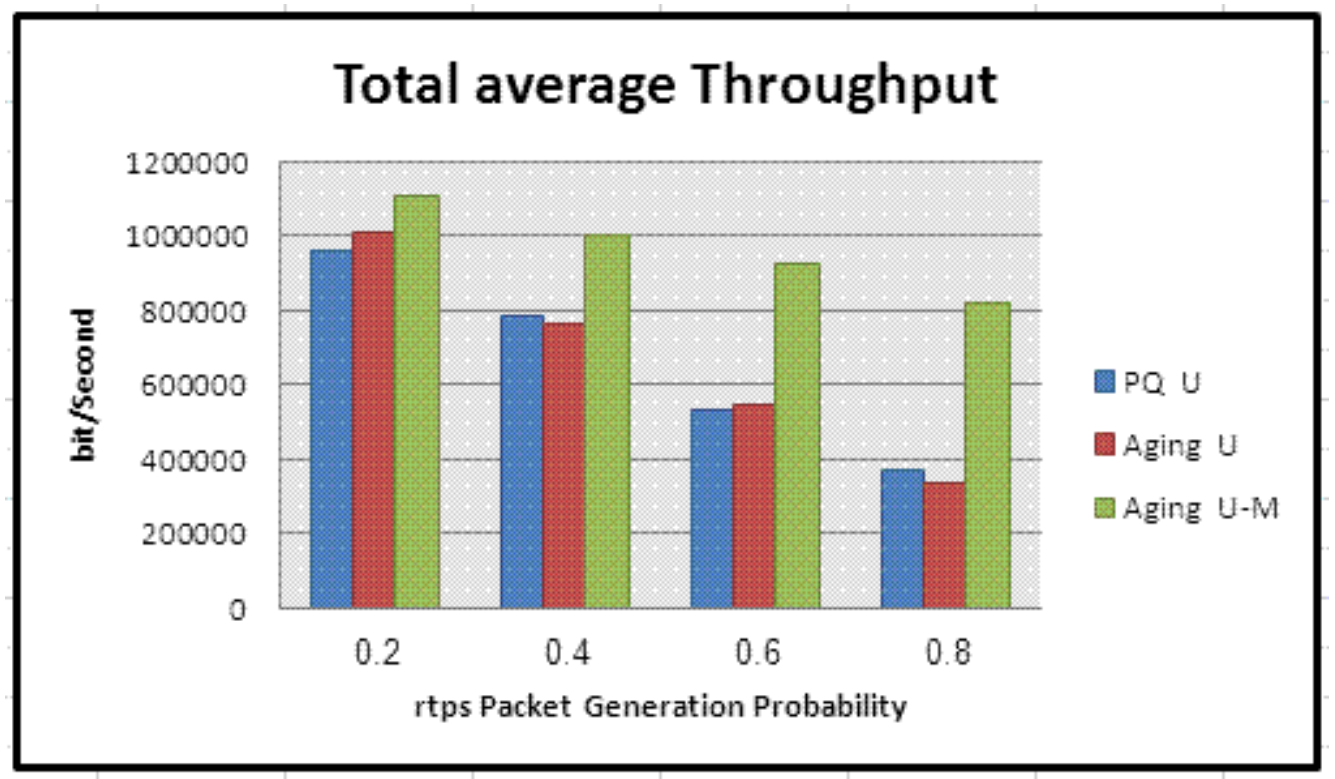

Fig. 12. Total Average Throughput

\section{Conclusion}

In this paper, we have proposed a two-level scheduling mechanism which is a combination of PQ, WRR and FCFS algorithms and Aging method. The simulation results conducted on this mechanism show that QoS requirements such as delay, throughput for low priority classes of service, especially BE can be met to the expected limit. Furthermore, by implementation of multicast mode in rtps class of service, we can guarantee the QoS requirements especially throughput and other classes of service delays and as a whole, increase the throughput of the system.

\section{References}

[1] IEEE Std 802.16e - 2005 and IEEE Std 802.16 - 2004/Corl - 2005.(Amendment and Corrigendum to IEEE Std 802.16 -2004).

[2] C.So-In , R.Jain,A.Tamimi " Scheduling in IEEE 802.16 mobile WiMAX networks key issues and a survey".

[3] Fen Hou, Pin-Han Ho, Xuemin (Sherman) Shen. "An efficient delay constrained scheduling scheme for IEEE 802.16 networks"Springer Science+Business Media, LLC 2007 .

[4] L.Nuaymi. "WiMAX : Technology for Broadband Wireless Access ".Jhon Wiley \& Sons 2007.

[5] Sheng-Tzong Cheng, Bo-Fu Chen, and Chih-Lun Chou "Fairness-based Scheduling Algorithm for TDD Mode IEEE 802.16 Broadband Wireless Access Systems “.2008.

[6] Vishal Sharma , Namita Vamancy "The Uniformly-Fair Deficit Round-Robin (UF-DRR) Scheduler for Improved QoS Guarantees in IEEE 802.16 WiMax Networks".2007.

[7] Yi Wu, Yanqun Le , Dongmei Zang "An Internal Scheduling Framework for Management of Best Effort Connections per SS in WiMAX System”.2008. 
[8] Xiaojuan Xie, Haining Chen Hongyi Wu "Simulation Studies of a Fair and Effective Queueing Algorithm for WiMAX Resource Allocation”.2008.

[9] Alexander Sayenko, Olli Alanen, Timo Hamalainen "Scheduling solution for the IEEE 802.16 base Station".2007.

[10] Mehri Mehrjoo, Mehrdad Dianati, Xuemin Shen, Kshirasagar Naik "Opportunistic fair scheduling for the downlink of IEEE 802.16 Wireless Metropolitan Area Networks".

[11] Nararat RUANGCHAIJATUPON , Yusheng JI "Adaptive Scheduling with Fairness in IEEE 802.16e Networks".2007. 